\title{
Having a Ball With the Sphere
}

\author{
Dirk Johan Stromberg ${ }^{*}$, Robert Casteels $^{\dagger}$ \\ *Department of Music, LASALLE College of the Art, Singapore \\ ${ }^{\dagger}$ Centre for the Arts, National University of Singapore \\ dirk.stromberg@lasalle.edu.sg
}

\begin{abstract}
Robert Casteels has composed a growing corpus of more than 100 musical works cross cultures, genres and disciplines. These range from miniature to large-scale works in the European tradition, as well as multidisciplinary works which combine European, Chinese and Indian instruments, as well as the gamelan, together with computer-generated sound and images. Instances of space for creative interaction between the performers in these compositions have been far and few between. The work, time:space:, has unveiled new possibilities for interaction and a blurring the line between performer and composer creating an artistic interconnectivity and interdependency.
\end{abstract}

Keywords: new instruments, composition, live performance

\section{Introduction}

A research grant from the National Arts Council (Singapore) enabled Casteels, together with electro-acoustician Dirk Stromberg and visual art director Andrew Thomas, to research and create two works, 2014:time:space: and 2015:time:space: (collectively referred to as $x x x x$ :timespace:). Each was a one-hour work with "e-instruments" such as the Sphere which is presented in this article. Both works also involved dance, human voice, and video in three superimposed layers: a pre-recorded soundtrack of electro-acoustic music, the sounds produced by the electronic instruments and the human voice, and thirdly the transformation of these sounds (Casteels, 2014).

\section{The Sphere}

The Sphere is an electronic instrument built by Stromberg in 2014. It was developed from the physical modeling work of Nicky Hind and Eric Lindemann, whose research looks at synthesis approaches and sound parameters. Nicky Hind describes various models for physical modeling which involve:
Articulation - the manner in which a sound is initiated, and:

Resonant body - the manner in which a sound propagates within the physical structure of the instrument.

This has then been described in synthesis terms as:

Excitation source - the initial source of a sound (i.e. a model of a blown or bowed sound);

Spectral characteristics - The resonance of sound that models the behavior of different instrumental shapes and bodies (Hind, 2015).

As suggested by Lindemann, the sonic parameters for synthesis and physical models can be simplified as:

Spectral skew - the tuning of partials;

Spectral parity - the balance between even and odd partials; 
Spectral tilt - the emphasis on either high or low partials and the degree of difference between the high and low partials;

Spectral angle - a subcategory of spectral tilt, the rate at which partials decay (Lindemann, 2015).

The Sphere was created with these concepts of physical modeling in mind. The goal was to create an instrument that allows for a performer to sculpt the sound through physical means. The choice of pressure sensors articulated with the fingers requires for the performer to use force in the creation of the sound. This is akin to the need for a wind player to blow and a string player to bow at different pressures to change register and color. The accelerometers require the player to use the wrists to orient the two halves of the sphere to further sculpt the sound, not unlike the changing of the angle of a bow or a plectrum. Keeping in line with the instrumental modeling, each parameter is interconnected. An example of this is the interdependent mapping of various aspects of amplitude, timbre and pitch. Figure 1 shows the layout and types of sensors, as well as the parameters they control, and Figure 2 shows the instrument in action.

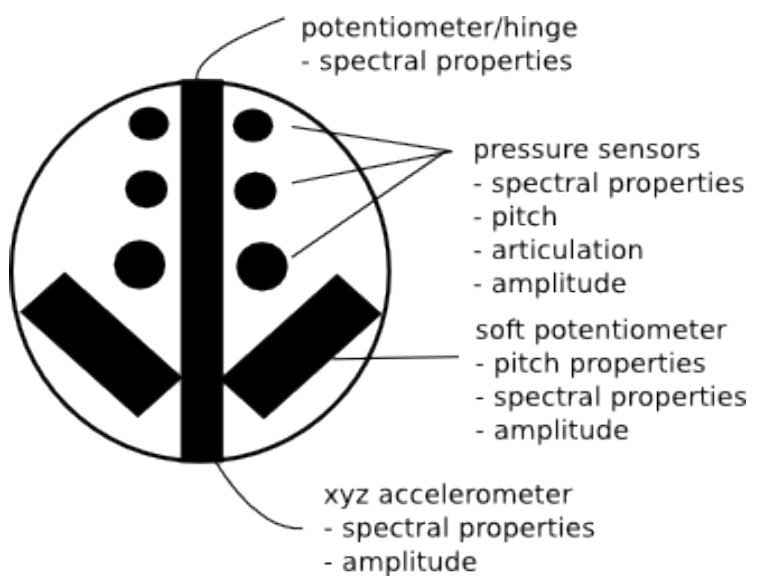

Figure 1. Diagram of the Sphere.

\section{The Sphere in $x x x x: t i m e: s p a c e:$ and its interconnectivity}

The musicians (on performing on the Sphere and singers) produced sounds. These sounds were processed, transformed and re- distributed in the performance space in real time via a 22-speaker diffusion system with a custom software built on psychoacoustic principles. The musicians reacted to these transformed and spatially distributed sounds.

The musicians depended on the sound engineers to control the dynamics, the timbral transformation and the spatial distribution of the sounds from the mixing desks. The sound engineers depended on the sounds produced by the musicians. In summary: in order to function as a composer and performer in the new sonic and visual environment of the xxxx:time:space: project, Casteels had to adapt his tactile pianistic sense to the sensitivity of the ten sensors on The Sphere, had to relinquish levels of control to sound engineers and had to change the type and level of interactivity with fellow performers.

The traditional labor division between composers, performers and engineers is blurred. Already in 1936, French composer Edgar Varèse noted the solidarity between scientific development and the progress of music when he declared: "I dream of instruments obedient to my thought and which with their contribution of a whole new world of unsuspected sounds, will lend themselves to the exigencies of my inner rhythm" (Schwartz, 1998).

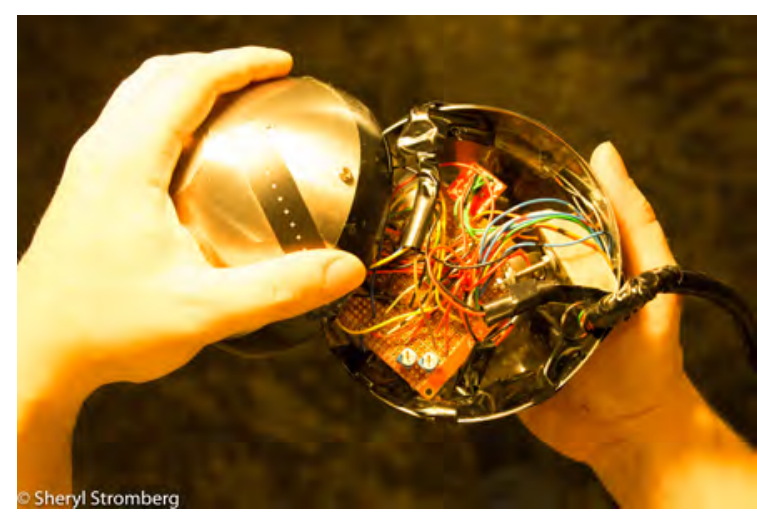

Figure 2. Manipulating the Sphere.

\section{The score}

The Sphere player and singers not only reacted to sounds but also anticipated sonic events of the pre-recorded track thanks to a score visible on a computer screen. This score consists of a waveform. Performers and engineers also had to relate to the images that 
were projected on four screens (Casteels, 2014). Figure 3 shows the cover of the score.

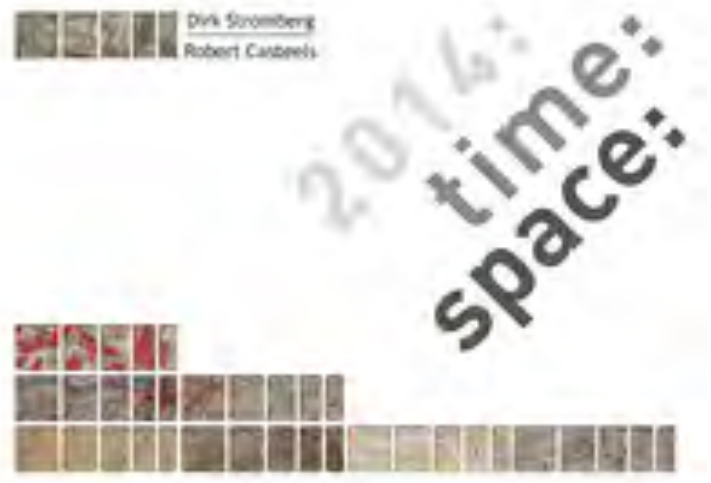

Figure 3. Cover of the score of 2014:time:space

\section{Further development}

The Sphere was a stepping-stone in the approach of instrument development through the exploration of tactile feedback and its musical implementation in various synthesizes. The use of pressure sensors was successful because an acceptable level of expressiveness and interconnection between performer and instrument was reached. The physical structure of the instrument was frail and the reapplication of the instrument is limited. The outcome is to develop a more sturdy and universal instrument de- sign system that allows for instrumentalists to create instruments, rather than impose a design on a performer.

\section{References}

Casteels, R. (2002, February 12). URL Robercasteels.com. Retrieved July 2, 2015.

Casteels, R. (2014, October 15). Xxxx:time:space:. Retrieved July 2, 2015, from URL http://timespace.sg

Casteels, R. (2014, December 10). 2014:time:space:. Singapore: Casteels.

Hind, N. (n.d.) Physical Modeling Synthesis. Retrieved July 31, 2015, from URL https://ccrma.stanford.edu/software/clm/compmus /clm-tutorials/pm.html.

Lindemann, E. (n.d.) Synful Orchestra Users Guide. Retrieved July 31, 2015, from URL http://www.synful.com/Synful_Orchestra_Users_G vide.pdf.

The Liberation of Sound. (1998). In E. Schwartz \& B. Childs (Eds.), Contemporary Composers on Contemporary Music (Expanded Edition ed., P. 196). New York, New York: Da Cap Press.

Thomas, A. [creativehybrid]. (7 March 2015). 2014:time:space: Premiere Live Performance [video file]. Retrieved from URL

https://youtu.be/glZkvs3XZVA. 